\title{
DIFFERENTIAL VARIATIONS IN BALLISTICS, WITH APPLICATIONS TO THE QUALITATIVE PROPER- TIES OF THE TRAJECTORY *
}

BY

\section{T. H. GRONWALL}

The investigation of the influence on the trajectory of changes in initial velocity, atmospheric conditions, etc., is most conveniently performed by the method of differential variations. These satisfy a system of linear differential equations of the fourth order, which was first set up in its most general form by F. R. Moulton. By the systematic use of the system of differential equations adjoint to the preceding one, Bliss has developed a very simple and practical method for computing all the differential variations. $\dagger$

This method makes use of the existence of a first integral $\lambda=$ const. (for explanation of notations, see section 1) by means of which the adjoint system reduces from the fourth to the third order. In the present paper, another first integral $x^{\prime} \lambda+y^{\prime} \mu+x^{\prime \prime} \nu+y^{\prime \prime} \rho=$ const. is established, and the adjoint system thereby reduced to the second order (sections 1-3), and in consequence, the numerical computation of the variations is materially shortened.

Of greater theoretical interest is the fact that, the system of linear differential equations involved being of the second order, the general behavior of their solutions may be determined in a fairly complete manner. This being done, a large number of qualitative properties of the trajectory follow almost immediately (sections 4 and 5). In the last section, attention is called to some unsolved problems of the same kind.

\section{The differential equations for the variations, and the adjoint SYSTEM}

The retardation (in the direction of the tangent to the trajectory) of a projectile moving with velocity $v$ through air of normal density is taken to be $v G(v) / C$, where the positive function $G(v)$ depends on $v$ alone and is the

* Presented to the Society, Oct. 25, 1919.

† G. A. Bliss: I. A method of computing differential corrections for a trajectory, J o u r n a l U. S. A r till e r y, vol. 51 (1919), p. 445. II. Differential equations containing arbitrary functions, these Tr a $\mathrm{n}$ s a c.t i o n s, vol. 21 (1920), pp. 79-92. III. Functions of lines in ballistics, these Tran s a c t i o n s, vol. 21 (1920), pp. 93-106. 
same for all projectiles, while the ballistic coefficient $C$ depends on the weight and shape of the projectile. The decrease in atmospheric density with increasing altitude $y$ is taken into account by multiplying the previous expression for the retardation by a positive factor $H(y)$ where $H(0)=1$. $^{*}$

Referring the projectile to a right-handed system of rectangular coördinates $x, y, z$ with the origin at the muzzle of the gun, the positive $x$-axis horizontal and directed toward the target, the positive $y$-axis vertical and directed upward, so that the positive $z$-axis is directed to the right of the line of fire. the differential equations of the trajectory are $\dagger$

$$
\begin{aligned}
& x^{\prime \prime}=-E x^{\prime}, \\
& y^{\prime \prime}=-E y^{\prime}-g, \\
& z^{\prime \prime}=-E z^{\prime},
\end{aligned}
$$

where $g$ is the acceleration of gravity,

and

$$
E=\frac{G(v) H(y)}{C}
$$

The initial conditions are for $t=0$,

$$
\begin{array}{ll}
x_{0}=0, & x_{0}^{\prime}=v_{0} \cos \alpha, \\
y_{0}=0, & y_{0}^{\prime}=v_{0} \sin \alpha, \\
z_{0}=0, & z_{0}^{\prime}=0,
\end{array}
$$

where $v_{0}$ is the initial velocity and $\alpha$ the angle of departure. Since $\left(z^{\prime} / x^{\prime}\right)^{\prime}=0$ by (1), it follows from (4) that $z=0$.

Let us now consider the variations in this trajectory introduced by changes $\delta v_{0}$ in the initial velocity and $\delta \alpha$ in the angle of departure, or by changes in the retardation due to wind, to changes in the functions $G(v)$ and $H(y)$ produced by other atmospheric conditions, and to a change in the ballistic coefficient $C$. Denote by $G_{1}(v)=G(v)+\delta G(v), H_{1}(y)=H(y)+\delta H(y), C_{1}=C+\delta C$ the new $G$ - and $H$-functions and the new ballistic coefficient, and the components of the wind velocity $w$, which we assume to depend on the altitude $y$ alone, by $w_{x}=w_{x}(y), w_{y}=w_{y}(y), w_{z}=w_{z}(y)$. Let the coördinates of the projectile in the changed trajectory be $\bar{x}, \bar{y}, \bar{z}$ at the time $t$; the retardation of the projectile, at a given altitude $\bar{y}$, depends only on its velocity $V$

* In practice, $H(y)=e^{-0.0001025 y}$ (lengths being measured in meters and time in seconds), while $G(v)$ is given in tabular form.

$\uparrow$ The notations used, which differ in some respects from those of Bliss, are those officially adopted by the Technical Staff. 
relative to the atmosphere, the components of which are $\bar{x}^{\prime}-w_{x}(\bar{y})$, $\bar{y}^{\prime}-w_{y}(\bar{y}), \bar{z}^{\prime}-w_{z}(\bar{y})$, so that the expression for the retardation now becomes

$$
\frac{V G_{1}(V) H_{1}(\bar{y})}{C_{1}}=V E_{1}
$$

Consequently, the differential equations of the changed $t$ ajectory become

$$
\begin{aligned}
& \bar{x}^{\prime \prime}=-E_{1}\left(\bar{x}^{\prime}-w_{x}(\bar{y})\right), \\
& \bar{y}^{\prime \prime}=-E_{1}\left(\bar{y}^{\prime}-w_{y}(\bar{y})\right)-g, \\
& \bar{z}^{\prime \prime}=-E_{1}\left(\bar{z}^{\prime}-w_{z}(\bar{y})\right),
\end{aligned}
$$

with the initial conditions

Now write

$$
\begin{array}{ll}
\bar{x}_{0}=0, & \bar{x}_{0}^{\prime}=\left(v_{0}+\delta v_{0}\right) \cos (\alpha+\delta \alpha), \\
\bar{y}_{0}=0, & \bar{y}_{0}^{\prime}=\left(v_{0}+\delta v_{0}\right) \sin (\alpha+\delta \alpha), \\
\bar{z}_{0}=0, & \bar{z}_{0}^{\prime}=0 .
\end{array}
$$

$$
\Delta x=\bar{x}-x, \quad \Delta y=\bar{y}-y, \quad \Delta z=\bar{z}-z
$$

and subtract equations (1) from (6); we obtain

$$
\begin{aligned}
(\Delta x)^{\prime \prime}=-E\left[(\Delta x)^{\prime}-w_{x}\right. & (y+\Delta y)] \\
& -\left(E_{1}-E\right)\left[x^{\prime}+(\Delta x)^{\prime}-w_{x}(y+\Delta y)\right], \\
(\Delta y)^{\prime \prime}=-E\left[(\Delta y)^{\prime}-w_{y}\right. & (y+\Delta y)] \\
& -\left(E_{1}-E\right)\left[y^{\prime}+(\Delta y)^{\prime}-w_{y}(y+\Delta y)\right], \\
(\Delta z)^{\prime \prime}=-E\left[(\Delta z)^{\prime}-w_{z}\right. & (y+\Delta y)] \\
& -\left(E_{1}-E\right)\left[z^{\prime}+(\Delta z)^{\prime}-w_{z}(y+\Delta y)\right],
\end{aligned}
$$

with the initial conditions for $t=0$

$$
\begin{array}{ll}
(\Delta x)_{0}=0, & (\Delta x)_{0}^{\prime}=\left(v_{0}+\delta v_{0}\right) \cos (\alpha+\delta \alpha)-v_{0} \cos \alpha, \\
(\Delta y)_{0}=0, & (\Delta y)_{0}^{\prime}=\left(v_{0}+\delta v_{0}\right) \sin (\alpha+\delta \alpha)-v_{0} \sin \alpha, \\
(\Delta z)_{0}=0, & (\Delta z)_{0}^{\prime}=0 .
\end{array}
$$

We shall assume that, for all values of $t$, we have

$$
\left|w_{x}(y+\Delta y)-w_{x}(y)\right|<c W_{x} \cdot \Delta y
$$

where $c$ is a constant and $W_{x}$ equals the maximum value of $w_{x}(y)$ along the trajectory, ${ }^{*}$ with similar conditions on $w_{y}$ and $w_{z}$, and moreover that $G(v)$

*This assumption is introduced in order to make the difference $w_{x}(y+\Delta y)-w_{x}(y)$ a small quantity of the second order in the expansions below. 
and $H(y)$ have bounded derivatives of the second order. We now expand the differences to the right in (9) by Taylor's theorem with remainder term of the second order, and denote by dots any terms containing as factors squares or products of the following quantities: $\Delta x, \Delta y, \Delta z,(\Delta x)^{\prime},(\Delta y)^{\prime}$, $(\Delta z)^{\prime}, w_{x}(y), w_{y}(y), w_{z}(y), \delta G(v), \delta G^{\prime}(v)\left[=G_{1}^{\prime}(v)-G^{\prime}(v)\right], \delta H(y)$, $\delta H^{\prime}(y)$ and $\delta C$. We thus obtain

$$
(\Delta x)^{\prime}-w_{x}(y+\Delta y)=(\Delta x)^{\prime}-w_{x}(y)+\cdots
$$

and similar expressions in $y$ aid $z$; moreover, writing $w_{x}, w_{y}, w_{z}$ for $w_{x}(y)$, $w_{y}(y), w_{z}(y)$ and observing that $z^{\prime}=0$,

$$
\begin{aligned}
& V^{2}=\left[x^{\prime}+(\Delta x)^{\prime}-w_{x}(y+\Delta y)\right]^{2} \\
& +\left[y^{\prime}+(\Delta y)^{\prime}-w_{y}(y+\Delta y)\right]^{2} \\
& +\left[(\Delta z)^{\prime}-w_{z}(y+\Delta y)\right]^{2} \\
& =v^{2}+2 x^{\prime}\left[(\Delta x)^{\prime}-w_{x}\right]+2 y^{\prime}\left[(\Delta y)^{\prime}-w_{y}\right]+\cdots ; \\
& V=v+\frac{x^{\prime}}{v}\left[(\Delta x)^{\prime}-w_{x}\right]+\frac{y^{\prime}}{v}\left[(\Delta y)^{\prime}-w_{y}\right]+\cdots ; \\
& G_{1}(V)=G_{1}(v)+(V-v) G_{1}^{\prime}(v)+\cdots \\
& =G(v)+\delta G(v)+(V-v) G^{\prime}(v)+\cdots ; \\
& H_{1}(\bar{y})=H_{1}(y)+\Delta y \cdot H_{1}^{\prime}(y)+\cdots \\
& =H(y)+\delta H(y)+\Delta y \cdot H^{\prime}(y)+\cdots ; \\
& \frac{1}{C_{1}}=\frac{1}{C+\delta C}=\frac{1}{C}\left(1-\frac{\delta C}{C}+\cdots\right) ; \\
& \frac{G_{1}(V) H_{1}(\bar{y})}{C_{1}}=\frac{G(v) H(y)}{C}\left[1+\frac{\delta G(v)}{G(v)}+(V-v) \frac{G^{\prime}(v)}{G(v)}\right. \\
& \left.+\frac{\delta H(y)}{H(y)}+\Delta y \cdot \frac{H^{\prime}(y)}{H(y)}-\frac{\delta C}{C}+\cdots\right]
\end{aligned}
$$

and replacing $V-v$ by (12)

$$
\begin{aligned}
& E_{1}-E=E\left\{\frac{x^{\prime}\left[(\Delta x)^{\prime}-w_{x}\right]+y^{\prime}\left[(\Delta y)^{\prime}-w_{y}\right]}{v} \frac{G^{\prime}(v)}{G(v)}\right. \\
& \left.+\Delta y \frac{H^{\prime}(y)}{H(y)}+\frac{\delta G(v)}{G(v)}+\frac{\delta H(y)}{H(y)}-\frac{\delta C}{C}+\cdots\right\} .
\end{aligned}
$$

We now introduce (11) and (12) in (9), replace $\Delta x, \Delta y$ and $\Delta z$ by $\xi, \eta$ and $\zeta$ and omit all the terms denoted by dots; writing $\xi^{\prime}=\xi_{1}$ and $\eta^{\prime}=\eta_{1}$, we thus obtain the differential equations 


$$
\begin{aligned}
& \xi^{\prime}=\quad \xi_{1} \\
& \eta^{\prime}=\quad \eta_{1} \\
& \xi_{1}^{\prime}=-x^{\prime} E \frac{H^{\prime}}{H} \eta-E\left(1+x^{\prime 2} \frac{G^{\prime}}{v G}\right) \xi_{1}-\quad x^{\prime} y^{\prime} E \frac{G^{\prime}}{v G} \eta_{1} \\
& +E\left(1+x^{\prime 2} \frac{G^{\prime}}{v G}\right) w_{x}+x^{\prime} y^{\prime} E \frac{G^{\prime}}{v G} w_{\nu}-x^{\prime} E\left(\frac{\delta G}{G}+\frac{\delta H}{H}-\frac{\delta C}{C}\right) \\
& \eta_{1}^{\prime}=-y^{\prime} E \frac{H^{\prime}}{H} \eta-\quad x^{\prime} y^{\prime} E \frac{G^{\prime}}{v G} \xi_{1}-E\left(1+y^{\prime 2} \frac{G^{\prime}}{v G}\right) \eta_{1} \\
& +x^{\prime} y^{\prime} E \frac{G^{\prime}}{v G} w_{x}+E\left(1+y^{\prime 2} \frac{G^{\prime}}{v G}\right) w_{y}-y^{\prime} E\left(\frac{\delta G}{G}+\frac{\delta H}{H}-\frac{\delta C}{C}\right) ;
\end{aligned}
$$

(15) $\zeta^{\prime \prime}=-E\left(\zeta^{\prime}-w_{z}\right)$,

with the initial conditions for $t=0$, obtained from (7) by expanding and omitting powers of $\delta v_{0}$ and $\delta \alpha$ higher than the first,

$$
\begin{aligned}
\xi(0) & =0, \\
\eta(0) & =0, \\
\xi_{1}(0) & =\cos \alpha \cdot \delta v_{0}-v_{0} \sin \alpha \cdot \delta \alpha=x_{0}^{\prime} \frac{\delta v_{0}}{v_{0}}-y_{0}^{\prime} \delta \alpha, \\
\eta_{1}(0) & =\sin \alpha \cdot \delta v_{0}+v_{0} \cos \alpha \cdot \delta \alpha=y_{0}^{\prime} \frac{\delta v_{0}}{v_{0}}+x_{0}^{\prime} \delta \alpha, \\
\zeta(0) & =0, \quad \zeta^{\prime}(0)=0 .
\end{aligned}
$$

The quantities $\xi, \eta, \zeta$ defined by (14)-(17) are the differential variations (of the first order) of $x, y$ and $z$. This appellation is justified by the following property, for the proof of which see Bliss III:

Let $\epsilon$ be any positive quantity, and suppose that $\delta v_{0}, \delta \alpha, \delta C, w_{x}, w_{y}, w_{z}$, $\delta G, \delta G^{\prime}, \delta H$ and $\delta H^{\prime}$ are less than $\epsilon$ in absolute value (the last seven at every point of the trajectory). Then the expressions

$$
\frac{\Delta x-\xi}{\epsilon}, \quad \frac{(\Delta x)^{\prime}-\xi_{1}}{\epsilon}, \quad \frac{\Delta y-\eta}{\epsilon}, \quad \frac{(\Delta y)^{\prime}-\eta_{1}}{\epsilon}, \frac{\Delta z-\zeta}{\epsilon}, \frac{(\Delta z)^{\prime}-\zeta^{\prime}}{\epsilon}
$$

tend toward zero with $\epsilon$, and this uniformly in any time interval $0 \leqq t \leqq t_{0}$.

In integrating the equations for the differential variations, we begin with (15) and (17), which define the lateral deflection $\zeta(t)$ of the projectile at the time $t$.

From the first of (1), we have $E=-x^{\prime \prime} / x^{\prime}$, and (15) becomes

whence by (17)

$$
\left(\zeta^{\prime} / x^{\prime}\right)^{\prime}=E w_{z} / x^{\prime}
$$

$$
\zeta^{\prime}=x^{\prime} \int_{0}^{t} \frac{E w_{z}}{x^{\prime}} d t
$$


integrating again, we obtain

$$
\zeta(t)=\int_{0}^{t} E(\tau) \frac{x(t)-x(\tau)}{x_{\tau}^{\prime}} w_{z} d \tau,
$$

as is readily verified by differentiation, and in the particular case where $w_{z}=0$ for $\tau<t_{0}$, but $w_{z}=$ const. $\neq 0$ for $\tau \geqq t_{0}$, we háve

$$
\zeta(t)=w_{z} \int_{t_{0}}^{t}[x(t)-x(\tau)] d\left(\frac{1}{x^{\prime}(\tau)}\right) .
$$

Integrating by parts, we find the

Deflection due to a constant cross wind $w_{2}$ from the time to onward

$$
\zeta(t)=\left[t-t_{0}-\frac{x(t)-x\left(t_{0}\right)}{x^{\prime}\left(t_{0}\right)}\right] w_{z} .
$$

To obtain expressions for $\xi, \eta$, $\xi_{1}$ and $\eta_{1}$, we follow Bliss in introducing the system of linear differential equations adjoint to the system (14), namely

$$
\begin{aligned}
\lambda^{\prime} & =0, \\
\mu^{\prime} & =\frac{H^{\prime}}{H} E\left(x^{\prime} \nu+y^{\prime} \rho\right), \\
\nu^{\prime} & =-\lambda+E \nu+x^{\prime} E \frac{G^{\prime}}{v G}\left(x^{\prime} \nu+y^{\prime} \rho\right), \\
\rho^{\prime} & =-\mu+E \rho+y^{\prime} E \frac{G^{\prime}}{v G}\left(x^{\prime} \nu+y^{\prime} \rho\right) .
\end{aligned}
$$

By the fundamental property of the adjoint system, any solution $\xi, \eta, \xi_{1}, \eta_{1}$ of (14) and any solution $\lambda, \mu, \nu, \rho$ of (20) are connected by the relation

$$
\begin{aligned}
\left(\xi \lambda+\eta \mu+\xi_{1} \nu+\eta_{1} \rho\right)^{\prime}= & {\left[E\left(1+x^{\prime 2} \frac{G^{\prime}}{v G}\right) w_{x}+x^{\prime} y^{\prime} E \frac{G^{\prime}}{v G} w_{\nu}\right.} \\
& \left.-x^{\prime} E\left(\frac{\delta G}{G}+\frac{\delta H}{H}-\frac{\delta C}{C}\right)\right] \nu \\
& +\left[x^{\prime} y^{\prime} E \frac{G^{\prime}}{v G} w_{x}+E\left(1+y^{\prime 2} \frac{G^{\prime}}{v G}\right) w_{y}\right. \\
& \left.-y^{\prime} E\left(\frac{\delta G}{G}+\frac{\delta H}{H}-\frac{\delta C}{C}\right)\right] \rho,
\end{aligned}
$$

or reducing the right-hand member by means of the last two equations (20)

$$
\begin{aligned}
\left(\xi \lambda+\eta \mu+\xi_{1} \nu+\eta_{1} \rho\right)^{\prime}=\left(\lambda+\nu^{\prime}\right) w_{x}+\left(\mu+\rho^{\prime}\right) w_{y} \\
\\
\quad-E\left(x^{\prime} \nu+y^{\prime} \rho\right)\left(\frac{\delta G}{G}+\frac{\delta H}{H}-\frac{\delta C}{C}\right)
\end{aligned}
$$


Integrating from $t=0$ to $t=t_{0}$ and using (16), we find

$$
\begin{aligned}
\left(\xi \lambda+\eta \mu+\xi_{1} \nu+\eta_{1} \rho\right)_{t=t_{0}}= & \left(x^{\prime} \nu+y^{\prime} \rho\right)_{0} \frac{\delta v_{0}}{v_{0}}+\left(x^{\prime} \rho-y^{\prime} \nu\right)_{0} \delta \alpha \\
& +\int_{0}^{t_{0}}\left(\lambda+\nu^{\prime}\right) w_{x} d t+\int_{0}^{t_{0}}\left(\mu+\rho^{\prime}\right) w_{\nu} d t \\
& -\int_{0}^{t_{0}} E\left(x^{\prime} \nu+y^{\prime} \rho\right)\left(\frac{\delta G}{G}+\frac{\delta H}{H}-\frac{\delta C}{C}\right) d t .
\end{aligned}
$$

This formula gives us the value of any linear combination of $\xi, \eta, \xi_{1}$ and $\eta_{1}$ at the time $t=t_{0}$, provided that we have obtained a solution of (20) taking as initial values for $t=t_{0}$ the coefficients of the linear combination in question.

2. First integrals of the adjoint system, aNd Reduction of the LATter FROM THE FOURTH TO THE SECOND ORDER

The existence of two first integrals of (20) is due to the fact that the equations (1) contain neither $x$ nor $t$ explicitly. Since the equations (1) do not contain $x$ explicitly, they are also satisfied by $\bar{x}=x+\epsilon, \bar{y}=y, \bar{z}=z$, where $\epsilon$ is a constant, or in other words, these $\bar{x}, \bar{y}, \bar{z}$ satisfy the particular equations (6) in which $w_{x}=w_{y}=w_{z}=\delta G=\delta H=\delta C=0$. We have here $\Delta x=\epsilon, \Delta y=(\Delta x)^{\prime}=(\Delta y)^{\prime}=0$, and the first order terms in respect to $\epsilon$ in these differences are $\xi=\epsilon, \eta=\xi_{1}=\eta_{1}=0$. These quantities must satisfy equations (14) (specialized of course to $w_{x}=\cdots=\delta C=0$ ), and consequently (21) specialized in the same way gives for any solution $\lambda, \mu, \nu, \rho$ of (20) upon division by $\epsilon,(1 \cdot \lambda+0 \cdot \mu+0 \cdot \nu+0 \cdot \rho)^{\prime}=0$ or $\lambda^{\prime}=0$, whence the first integral of (20).

$$
\lambda=\text { const. }
$$

This integral might obviously have been read off at once from the first of (20), but the derivation given emphasizes the analogy to the integral which we shall now obtain. Since equations (1) do not contain $t$ explicitly, they are also satisfied by $\bar{x}=x(t+\epsilon), \bar{y}=y(t+\epsilon), \bar{z}=z(t+\epsilon)$ with a constant $\epsilon$, and expanding the differences $\Delta x=x(t+\epsilon)-x(t),(\Delta x)^{\prime}=x^{\prime}(t+\epsilon)-x^{\prime}(t)$, etc., by Taylor's theorem, with remainder term of the second order, we see that the first order terms in $\epsilon$ are $\xi=x^{\prime}(t) \epsilon, \eta=y^{\prime}(t) \epsilon, \xi_{1}=x^{\prime \prime}(t) \epsilon$ and $\eta_{1}=y^{\prime \prime}(t) \epsilon$. These must satisfy the system (14) specialized as above, whence it follows as before from (21) that for every solution $\lambda, \mu, \nu, \rho$ of (20), we have $\left(x^{\prime} \lambda+y^{\prime} \mu+x^{\prime \prime} \nu+y^{\prime \prime} \rho\right)^{\prime}=0$ or

$$
\kappa+x^{\prime} \lambda+y^{\prime} \mu+x^{\prime \prime} \nu+y^{\prime \prime} \rho=0
$$

which is another first integral of $(20) .^{*}$ This integral may of course be verified by differentiating (24), substituting the values of $\lambda^{\prime}, \mu^{\prime}, \nu^{\prime}, \rho^{\prime}$ from (20) and

\footnotetext{
* It seems plausible that in general, that is, without specializing $G(v)$ or $H(y)$, there
} 
using the following formulas, obtained by differentiating (1) and (2),

$$
\begin{gathered}
x^{\prime \prime \prime}=\left(E^{2}-\frac{d E}{d t}\right) x^{\prime}, \quad y^{\prime \prime \prime}=\left(E^{2}-\frac{d E}{d t}\right) y^{\prime}+g \dot{E}, \\
\frac{1}{E} \frac{d E}{d t}=\frac{G^{\prime}}{G} v^{\prime}+\frac{H^{\prime}}{H} y^{\prime}=-E \frac{v G^{\prime}}{G}-g \frac{G^{\prime}}{v G} y^{\prime}+\frac{H^{\prime}}{H} y^{\prime},
\end{gathered}
$$

the last expression following from $v v^{\prime}=x^{\prime} x^{\prime \prime}+y^{\prime} y^{\prime \prime}=-E v^{2}-g y^{\prime}$. To perform the reduction of $(20)$ to the second order by means of the two integrals (23) and (24), we observe that (1) allows us to write the last two equations (20) in the forms

$$
\begin{aligned}
& \left(x^{\prime} \nu\right)^{\prime}=-x^{\prime} \lambda+x^{\prime 2} E \frac{G^{\prime}}{v G}\left(x^{\prime} \nu+y^{\prime} \rho\right), \\
& \left(y^{\prime} \nu\right)^{\prime}=-y^{\prime} \lambda-g \nu+x^{\prime} y^{\prime} E \frac{G^{\prime}}{v G}\left(x^{\prime} \nu+y^{\prime} \rho\right), \\
& \left(x^{\prime} \rho\right)=-x^{\prime} \mu+x^{\prime} y^{\prime} E \frac{G^{\prime}}{v G}\left(x^{\prime} \nu+y^{\prime} \rho\right), \\
& \left(y^{\prime} \rho\right)^{\prime}=-y^{\prime} \mu-g \rho+y^{\prime 2} E \frac{G^{\prime}}{v G}\left(x^{\prime} \nu+y^{\prime} \rho\right),
\end{aligned}
$$

whence

$$
\left(x^{\prime} \nu+y^{\prime} \rho\right)^{\prime}=-x^{\prime} \lambda-y^{\prime} \mu-g \rho+E \frac{v G^{\prime}}{G}\left(x^{\prime} \nu+y^{\prime} \rho\right) .
$$

On the other hand, the substitution of $x^{\prime \prime}$ and $y^{\prime \prime}$ from (1) in (24) gives

$$
g \rho=\kappa+x^{\prime} \lambda+y^{\prime} \mu-E\left(x^{\prime} \nu+y^{\prime} \rho\right) .
$$

Introducing the notation

$$
h=h(y)=-\frac{H^{\prime}(y)}{H(y)},
$$

and writing down the second of (20) together with the result of substituting $g \rho$ from (27) in the preceding expression for $\left(x^{\prime} \nu+y^{\prime} \rho\right)^{\prime}$, we find

$$
\begin{aligned}
\mu^{\prime} & =-h E\left(x^{\prime} \nu+y^{\prime} \rho\right), \\
\left(x^{\prime} \nu+y^{\prime} \rho\right)^{\prime} & =\left(1+\frac{v G^{\prime}}{G}\right) E\left(x^{\prime} \nu+y^{\prime} \rho\right)-2\left(x^{\prime} \lambda+y^{\prime} \mu\right)-\kappa,
\end{aligned}
$$

which is the required second order system in $\mu$ and $x^{\prime} \nu+y^{\prime} \rho$. Having exists no further integral of (20) algebraic in $\lambda, \mu, \nu, \rho, x, y$ and their derivatives. In special cases, however, new integrals may be found. Thus, for $H(y)=$ const. (corresponding to an atmosphere of constant density) the second equation (20) gives $\mu=$ const., while in the case $G(v)=$ const. (so that the retardation at normal air density is proportional to the velocity) the third equation (20) combined with (23) gives $x^{\prime} \nu+x \lambda=$ const. 
integrated (29), we obtain $\rho$ from (27) and $\nu$ from the identity:

$$
\nu=\frac{1}{x^{\prime}}\left(x^{\prime} \nu+y^{\prime} \rho\right)-\frac{y^{\prime}}{x^{\prime}} \rho . *
$$

Before proceeding further, we note that (26) gives

$$
\left(x^{\prime} \rho-y^{\prime} \nu\right)^{\prime}=y^{\prime} \lambda-x^{\prime} \mu+g \nu
$$

and that, by means of (1) and (25), the second equation (29) may be written in the form

$$
\begin{aligned}
& {\left[x^{\prime} E\left(x^{\prime} \nu+y^{\prime} \rho\right)\right]^{\prime}} \\
& \quad=-\left(h+g \frac{G^{\prime}}{v G}\right) x^{\prime} y^{\prime} E\left(x^{\prime} \nu+y^{\prime} \rho\right)-x^{\prime} E\left(2 x^{\prime} \lambda+2 y^{\prime} \mu+\kappa\right) .
\end{aligned}
$$

\section{Formulas For the variations in RANGe AND MaXimum oRdinate}

In the following, coördinates, velocities and times pertaining to the point of fall (where $y=0$ ) will be denoted by the subscript $\omega$, and those pertaining to the summit (where $y^{\prime}=0$ ) by the subscript $s$.

On the trajectory defined by $x=x(t), y=y(t)$ the time of fight $t_{\omega}$ is the positive root of the equation

and the range $x_{\omega}$ is given by

$$
y\left(t_{\omega}\right)=0,
$$

$$
x_{\omega}=x\left(t_{\omega}\right) \cdot \dagger
$$

The angle of fall $\omega$ is the angle between 0 and $\pi / 2$ defined by

$$
\tan \omega=-\frac{y_{\omega}^{\prime}}{x_{\omega}^{\prime}}=-\frac{y^{\prime}\left(t_{\omega}\right)}{x^{\prime}\left(t_{\omega}\right)}
$$

The time $t_{s}$ required to reach the summit of the trajectory is determined by the equation

$$
y^{\prime}\left(t_{s}\right)=0
$$

and the maximum ordinate is then

$$
y_{s}=y\left(t_{s}\right) .
$$

In the changed trajectory defined by $\bar{x}=x(t)+\Delta x(t), \bar{y}=y(t)+\Delta y(t)$,

* The system (29) is adapted to the theoretical purposes of the present paper. For the application of the method to the actual computation of differential variations, it is preferable to integrate numerically the differential equation of the second order in $\mu$ obtained by eliminating $x^{\prime} \nu+y^{\prime} \rho$ in (29). A detailed exposition of this method will be published elsewhere.

$\dagger$ For a proof of the existence of a unique positive root of (33), under the sole assumption that $E>0$ everywhere, see T. H. Gronwall, Qualitative properties of the ballistic trajectory, Innals of Mathematics, ser. II, vol. 21 (1920), pp. 44-65. 
$\bar{z}=\Delta z(t)$, the time of flight $t_{\omega}+\Delta t_{\omega}$ is given by

$$
y\left(t_{\omega}+\Delta t_{\omega}\right)+\Delta y\left(t_{\omega}+\Delta t_{\omega}\right)=0 .
$$

Now $y\left(t_{\omega}+\Delta t_{\omega}\right)=y\left(t_{\omega}\right)+y^{\prime}\left(t_{1}\right) \Delta t_{\omega}$, where $t_{1}$ lies between $t_{\omega}$ and $t_{\omega}+\Delta t_{\omega}$, and since $y\left(t_{\omega}\right)=0$, (25) gives $\Delta t_{\omega}=-\Delta y\left(t_{\omega}+\Delta t_{\omega}\right) / y^{\prime}\left(t_{1}\right)$. Hence $\Delta t_{\omega}$ is of the order of magnitude of $\Delta y$, that $i$; of $\eta$, since the difference $\Delta y-\eta$ is of higher order of magnitude than $\eta$; consequently $\Delta y\left(t_{\omega}+\Delta t_{\omega}\right)$ $=\eta\left(t_{\omega}\right)+(\mathrm{a}$ term of higher order of magnitude), and the first order term in $\Delta t_{\omega}$, or the differential variation $\delta t_{\omega}$ in time of flight is given by

$$
\delta t_{\omega}=-\frac{\eta\left(t_{\omega}\right)}{y^{\prime}\left(t_{\omega}\right)} \cdot
$$

The change in range $\Delta x_{\omega}$ is given by

$$
\begin{aligned}
x_{\omega}+\Delta x_{\omega}= & x\left(t_{\omega}+\Delta t_{\omega}\right)+\Delta x\left(t_{\omega}+\Delta t_{\omega}\right) \\
= & x\left(t_{\omega}\right)+x^{\prime}\left(t_{\omega}\right) \Delta t_{\omega}+\xi\left(t_{\omega}\right) \\
& +\left[\frac{1}{2} x^{\prime \prime}\left(t_{1}\right)\left(\Delta t_{\omega}\right)^{2}+\Delta x\left(t_{\omega}+\Delta t_{\omega}\right)-\xi\left(t_{\omega}\right)\right]
\end{aligned}
$$

with $t_{1}$ between $t_{\omega}$ and $t_{\omega}+\Delta t_{\omega}$, the bracketed terms being of higher order of magnitude than the first. Retaining only terms of the first order of magnitude, we find the differential variation $\delta x_{\omega}$ in range to be $x^{\prime}\left(t_{\omega}\right) \delta t_{\omega}$ $+\xi\left(t_{\omega}\right)$, or substituting $\delta t_{\omega}$ from (39) and using (35)

$$
\delta x_{\omega}=\xi\left(t_{\omega}\right)+\eta\left(t_{\omega}\right) \cot \omega .
$$

The change in maximum ordinate $\Delta y_{\mathrm{s}}$ is given by

$$
y_{s}+\Delta y_{s}=y\left(t_{s}+\Delta t_{s}\right)+\Delta y\left(t_{s}+\Delta t_{s}\right),
$$

and since $y^{\prime}\left(t_{s}\right)=0$, the differences $y\left(t_{s}+\Delta t_{s}\right)-y\left(t_{s}\right)$ and $\Delta y\left(t_{s}+\Delta t_{s}\right)$ $-\eta\left(t_{s}\right)$ are of higher order of magnitude than the first, so that we obtain for the differential variation in maximum ordinate

$$
\delta y_{s}=\eta\left(t_{s}\right) \text {. }
$$

After these preliminaries, let us return to the remark at the end of section 2. By (40), $\delta x_{\omega}$ equals the linear combination $\xi \cdot 1+\eta \cdot \cot \omega+\xi_{1} \cdot 0+\eta_{1} \cdot 0$ at $t$ $=t_{\omega}$, and to obtain $\delta x_{\omega}$, we have therefore to integrate (20) with the initial conditions

$$
\lambda=1, \quad \mu=\cot \omega, \quad \nu=0, \quad \rho=0 \quad \text { for } \quad t=t_{\omega} .
$$

Substitution of $t=t_{\omega}$ in (23) therefore gives $\lambda=1$, and in (24),

$$
\kappa+x_{\omega}^{\prime}+y_{\omega}^{\prime} \cot \omega=0
$$

* For the general theorem of which this determination of $\delta t_{\omega}$ is a special application, see Bliss II, pp. 90-92. 
or $k=0$ by (35). Consequently, for the determination of the differential variations in range, the system (29) becomes

$$
\begin{aligned}
\mu^{\prime} & =-h E\left(x^{\prime} \nu+y^{\prime} \rho\right), \\
\left(x^{\prime} \nu+y^{\prime} \rho\right)^{\prime} & =\left(1+\frac{v G^{\prime}}{G}\right) E\left(x^{\prime} \nu+y^{\prime} \rho\right)-2\left(x^{\prime}+y^{\prime} \mu\right)
\end{aligned}
$$

with the initial conditions

$$
\mu=\cot \omega, x^{\prime} \nu+y^{\prime} \rho=0 \text { for } t=t_{\omega} .
$$

Moreover, (27) becomes

$$
g \rho=x^{\prime}+y^{\prime} \mu-E\left(\dot{x}^{\prime} \nu+y^{\prime} \rho\right),
$$

and (22), making $t_{0}=t_{\omega}$ and using (40)

$$
\begin{aligned}
\delta x_{\omega}= & \left(x^{\prime} \nu+y^{\prime} \rho\right)_{0} \frac{\delta v_{0}}{v_{0}}+\left(x^{\prime} \rho-y^{\prime} \nu\right)_{0} \delta \alpha \\
& +\int_{0}^{t_{\omega}}\left(1+\nu^{\prime}\right) w_{x} d t+\int_{0}^{t_{\omega}}\left(\mu+\rho^{\prime}\right) w_{y} d t \\
& -\int_{0}^{t_{\omega}} E\left(x^{\prime} \nu+y^{\prime} \rho\right)\left(\frac{\delta G}{G}+\frac{\delta H}{H}-\frac{\delta C}{C}\right) d t .
\end{aligned}
$$

Considering separately the various terms to the right in (46), we find the following expressions for:

Range variation due to change $\delta v_{0}$ in initial velocity:

$$
\delta x_{\omega}=\left(x^{\prime} \nu+y^{\prime} \rho\right)_{0} \frac{\delta v_{0}}{v_{0}} .
$$

Range variation due to change $\delta \alpha$ in angle of departure:

$$
\delta x_{\omega}=\left(x^{\prime} \rho-y^{\prime} \nu\right)_{0} \delta \alpha \text {. }
$$

Range variation due to a following wind $w_{x}$ :

$$
\delta x_{\omega}=\int_{0}^{t_{\omega}}\left(1+\nu^{\prime}\right) w_{x} d t
$$

and when $w_{x}=0$ for $t<t_{0}, w_{x}=$ const. $\neq 0$ for $t \geqq t_{0}$ :

Range variation due to a constant following wind $w_{x}$ from the time $t_{0}$ onward:

$$
\delta x_{\omega}=\left[t_{\omega}-t_{0}-\nu\left(t_{0}\right)\right] w_{x}
$$

(since $\nu\left(t_{\omega}\right)=0$ by (42)), and similarly

Range variation due to a constant vertical wind $w_{y}$ from the time $t_{0}$ onward:

$$
\delta x_{\omega}=\left[\int_{t_{0}}^{t_{\omega}} \mu d t-\rho\left(t_{0}\right)\right] w_{\nu} .
$$


Range variation due to a change $\delta H(y)$ in $H(y)$ :

$$
\delta x_{\omega}=-\int_{0}^{\omega_{\omega}} E\left(x^{\prime} \nu+y^{\prime} \rho\right) \frac{\delta H}{H} d t .
$$

Range variation due to a change $\delta C$ in the ballistic coefficient:

$$
\delta x_{\omega}=\frac{\delta C}{C} \int_{0}^{t_{\omega}} E\left(x^{\prime} \nu+y^{\prime} \rho\right) d t .
$$

Passing to the variations in maximum ordinate, (41) shows that $\delta y_{\mathrm{s}}$ equals the linear combination $\xi \cdot 0+\eta \cdot 1+\xi_{1} \cdot 0+\eta_{1} \cdot 0$ at $t=t_{s}$, and we have to integrate (20) with the initial conditions

$$
\lambda=0, \quad \mu=1, \quad \nu=0, \quad \rho=0 \quad \text { for } \quad t=t_{s} \cdot *
$$

Substitution of $t=t_{\mathrm{s}}$ in (23) therefore gives $\lambda=0$, and in (24), $\kappa=0$, since $y^{\prime}\left(t_{s}\right)=0$. Consequently, for the determination of the differential variations in maximum ordinate, the system (29) becomes

$$
\begin{aligned}
\mu^{\prime} & =-h E\left(x^{\prime} \nu+y^{\prime} \rho\right), \\
\left(x^{\prime} \nu+y^{\prime} \rho\right)^{\prime} & =\left(1+\frac{v G^{\prime}}{G}\right) E\left(x^{\prime} \nu+y^{\prime} \rho\right)-2 y^{\prime} \mu,
\end{aligned}
$$

with the initial conditions

$$
\mu=1, \quad x^{\prime} \nu+y^{\prime} \rho=0 \quad \text { for } \quad t=t_{s} .
$$

Moreover, (27) becomes

$$
g \rho=y^{\prime} \mu-E\left(x^{\prime} \nu+y^{\prime} \rho\right),
$$

and (22), making $t_{0}=t_{\text {a }}$ and using (41)

$$
\begin{aligned}
\delta y_{\imath}= & \left(x^{\prime} \nu+y^{\prime} \rho\right)_{0} \frac{\delta \dot{v}_{0}}{v_{0}}+\left(x^{\prime} \rho-y^{\prime} \nu\right)_{0} \delta \alpha \\
& +\int_{0}^{t_{0}} \nu^{\prime} w_{x} d t+\int_{0}^{t_{0}}\left(\mu+\rho^{\prime}\right) w_{\nu} d t \\
& -\int_{0}^{t_{0}} E\left(x^{\prime} \nu+y^{\prime} \rho\right)\left(\frac{\delta G}{G}+\frac{\delta H}{H}-\frac{\delta C}{C}\right) d t .
\end{aligned}
$$

Separating the terms to the right, we find for the

Maximum ordinate variation due to change $\delta v_{0}$ in initial velocity:

$$
\delta y_{\varepsilon}=\left(x^{\prime} \nu+y^{\prime} \rho\right)_{0} \frac{\delta v_{0}}{v_{0}},
$$

- The two sets of solutions of (20) belonging to range and maximum ordinate variations are thus entirely different, since they satisfy different initial conditions (42) and (53). This should be constantly borne in mind in the following, as we have refrained from distinguishing the two sets by subscripts in order to simplify the typography. 
Maximum ordinate variation due to change $\delta \alpha$ in angle of departure:

$$
\delta y_{s}=\left(x^{\prime} \rho-y^{\prime} \nu\right)_{0} \delta \alpha,
$$

Maximum ordınate variation due to a constant following wind $w_{x}$ from the time $t_{0}$ onward:

$$
\delta y_{\imath}=-\nu\left(t_{0}\right) w_{x},
$$

Maximum ordinate variation due to a constant vertical wind $w_{y}$ from the time $t_{0}$ onward:

$$
\delta y_{\bullet}=\left[\int_{t_{0}}^{t_{0}} \mu d t-\rho\left(t_{0}\right)\right] w_{y},
$$

Maximum ordinate variation due to a change $\delta H(y)$ in $H(y)$ :

$$
\delta y_{\iota}=-\int_{0}^{t_{\iota}} E\left(x^{\prime} \nu+y^{\prime} \rho\right) \frac{\delta H}{H} d t,
$$

Maximum ordinate variation due to a change $\delta C$ in the ballistic coefficient:

$$
\delta y_{s}=\frac{\delta C}{C} \int_{0}^{t_{s}} E\left(x^{\prime} \nu+y^{\prime} \rho\right) d t .
$$

4. Qualitative PROPERTIEs OF THE DIFFERential VARIATIONS IN RANGe

In addition to assuming $G(v)>0, H(y)>0$ as we have done from the outset in accordance with the physical significance of these functions, we shall introduce, in this and the following sections, the two hypotheses

$$
G^{\prime}(v)>0, \quad h(y) \geqq 0,
$$

the latter being equivalent to $H^{\prime}(y) \leqq 0$ by (28). Physically, this means that the retardation at normal density increases more rapidly than the first power of the velocity, and that the density of the atmosphere nowhere increases with the altitude.

The fundamental qualitative property of the range variations is expressed by the inequality

$$
x^{\prime} \nu+y^{\prime} \rho>0 \quad \text { for } \quad 0 \leqq t<t_{\omega} .
$$

To prove this, we write

$$
\psi=\int_{t}^{t \omega}\left(1+\frac{v G^{\prime}}{G}\right) E d t
$$

and obtain from the second equation (43) and (44)

$$
e^{\psi}\left(x^{\prime} \nu+y^{\prime} \rho\right)=2 \int_{0}^{t_{\omega}} e^{\psi}\left(x^{\prime}+y^{\prime} \mu\right) d t \text {. }
$$

Since $\left(x^{\prime}+y^{\prime} \mu\right)^{\prime}=-E\left(x^{\prime}+y^{\prime} \mu\right)-g \mu-y^{\prime} \mu^{\prime}$ by (1), and since at $t=t_{\omega}$ 
we have $x^{\prime}+y^{\prime} \mu=0$ by (35), $\mu=\cot \omega$ and $\mu^{\prime}=0$ by (43) and (44), it follows that $\left(x^{\prime}+y^{\prime} \mu\right)^{\prime}=-g \cot \omega$ at $t=t_{\omega}$, so that $\left(x^{\prime}+y^{\prime} \mu\right)^{\prime}<0$ for $t_{\omega}-\epsilon \leqq t \leqq t_{\omega}$, where $\epsilon$ is sufficiently small. Thus $x^{\prime}+y^{\prime} \mu$ decreases toward zero in this intervel, and (66) shows that $x^{\prime} \nu+y^{\prime} \rho$ is positive for $t_{\omega}-\epsilon<t<t_{\omega}$, or more generally, in the interior of any time interval ending at $t=t_{\omega}$, and in which $x^{\prime}+y^{\prime} \mu \geqq 0$. Now suppose that $x^{\prime} \nu+y^{\prime} \rho$ is not always positive in the interval $0 \leqq t<t_{\omega}$, then there exists a zero $t_{1}$ nearest to $t_{\omega}$ so that $x^{\prime} \nu+y^{\prime} \rho=0$ for $t=t_{1}$ but $x^{\prime} \nu+y^{\prime} \rho>0$ for $t_{1}<t<t_{\omega}$, and from what precedes it is seen that we must have

$$
x^{\prime}+y^{\prime} \mu<0 \text { for } t=t_{1} \text {. }
$$

The first of (43) shows that $\mu^{\prime} \leqq 0$ for $t_{1}<t<t_{w}$, and from (44), we consequently obtain

$$
\mu \geqq \cot \omega
$$

for $t_{1}<t<t_{\omega}$ at least. Since $x^{\prime}>0$ for all values of $t,{ }^{*}$ we conclude from (67) and (68) that $y^{\prime}\left(t_{1}\right)<0$, that is, the hypothetical zero of $x^{\prime} \nu+y^{\prime} \rho$ must occur, if at all, on the falling branch of the trajectory. For $t_{1}<t<t_{\omega}$, or more generally, as long as $y^{\prime}<0$ and $x^{\prime} \nu+y^{\prime} \rho>0$, it follows from the third of (26), the fundamental hypothesis (64), and (68) that

$$
\left(x^{\prime} \rho\right)^{\prime}<-x^{\prime} \mu \leqq-x^{\prime} \cot \omega,
$$

and integrating this inequality from $t$ to $t_{\omega}$ and observing that $\rho=0$ for $t=t_{\infty}$ by (42), we find

$$
x^{\prime} \rho>\left(x_{\omega}-x\right) \cot \omega
$$

for $t_{1} \leqq t<t_{\omega}$. But from (45) we obtain $g \rho=x^{\prime}+y^{\prime} \mu$ for $t=t_{1}$, since $x^{\prime} \nu+y^{\prime} \rho=0$ for this value of $t$, and in consequence of (67), $\rho$ must be negative at $t=t_{1}$, while (69) shows that $\rho$ is positive for this value of $t$, and this contradiction proves (65).

Moreover, it is now seen that (68) is true for $0 \leqq t<t_{\omega}$, the equality sign to be taken only when $h$ is identically zero, while (69) holds at least when $y^{\prime} \leqq 0$, that is, at least for $t_{\mathrm{s}} \leqq t<t_{\omega}$.

From the first of (43) and (65), we have $\mu^{\prime} \leqq 0$ for $0 \leqq t<t_{\omega}$, the equality sign to be taken only when $h=0$, and consequently, $\mu$ decreases toward cot $\omega$ as $t$ increases from 0 to $t_{\omega}$ (except when $h$ vanishes identically, $\mu$ being then equal to $\cot \omega$ ).

We shall now prove, moreover, that $x^{\prime}+y^{\prime} \mu$ is positive and decreases to zero when $t$ increases from 0 to $t_{\omega}$. On the rising branch of the trajectory where $y^{\prime}>0$, this is evident, since $x^{\prime}$ and $y^{\prime}$ are positive and decrease with $t$ increasing,

\footnotetext{
* This follows at once from $x_{0}^{\prime}=v_{0} \cos \alpha>0$, since (1) gives $x^{\prime}=x_{0}^{\prime} e \int_{0}^{t} E d t$.
} 
while $\mu$ is positive and decreases or remains constant. If $x^{\prime}+y^{\prime} \mu<0$ anywhere on the falling branch, we must have $x^{\prime}+y^{\prime} \mu=0$ and $\left(x^{\prime}+y^{\prime} \mu\right)^{\prime} \geqq 0$ at some point on the falling branch, since it was shown that $x^{\prime}+y^{\prime} \mu>0$ sufficiently near $t=t_{\omega}$. But at such a point $-g \mu-y^{\prime} \mu^{\prime} \geqq 0$ on account of

$$
\left(x^{\prime}+y^{\prime} \mu\right)^{\prime}=-E\left(x^{\prime}+y^{\prime} \mu\right)-g \mu-y^{\prime} \mu^{\prime},
$$

and this is impossible since $\mu>0, y^{\prime} \leqq 0, \mu^{\prime} \leqq 0$. Consequently $x^{\prime}+y^{\prime} \mu>0$ on the falling branch also (except at the point of fall, where it vanishes), and if $x^{\prime}+y^{\prime} \mu$ does not decrease steadily on the falling branch, there must be a minimum, distinct from the point of fall, and at the minimum point, $\left(x^{\prime}+y^{\prime} \mu\right)^{\prime}=0$ or $-E\left(x^{\prime}+y^{\prime} \mu\right)-g \mu-y^{\prime} \mu^{\prime}=\neq 0$, which is impossible, since the first two terms are negative, and the third negative or zero.

It is now possible to establish a number of qualitative properties of the differential variations in range.

Since, by (65), $x^{\prime} \nu+y^{\prime} \rho>0$ at $t=0$, it follows from (47) that The range increases when the initial velocity is increased.

Integrating the second of (43) from $t$ to $t_{\omega}$ and using (44), we find

$$
x^{\prime} \nu+y^{\prime} \rho=\int_{t}^{t \omega}\left[2\left(x^{\prime}+y^{\prime} \mu\right)-\left(1+\frac{v G^{\prime}}{G}\right) E\left(x^{\prime} \nu+y^{\prime} \rho\right)\right] d t,
$$

or integrating the term in $y^{\prime} \mu$ by parts and using the first of (43),

$$
x^{\prime} \nu+y^{\prime} \rho=2\left(x_{\omega}-x\right)-2 y \mu
$$

If we assume that

$$
-\int_{t}^{t_{\omega}}\left(1+\frac{v G^{\prime}}{G}-2 h y\right) E\left(x^{\prime} \nu+y^{\prime} \rho\right) d t .
$$

$$
2 h y<1+\frac{v G^{\prime}}{G}
$$

over the entire trajectory, the last term in (70) is negative by (65), and making $t=0$ so that $x=y=0$, we find $\left(x^{\prime} \nu+y^{\prime} \rho\right)_{0}<2 x_{\omega}$ or by (47), assuming $\delta v_{0}$ to be positive,

$$
\frac{\delta x_{\omega}}{x_{\omega}}<2 \frac{\delta v_{0}}{v_{0}}
$$

that is

For low trajectories, where $y$ is so small that (71) is satisfied everywhere, the range increases less rapidly than the square of the initial velocity.

When $h=0$, i.e., the atmospheric density is constant, (71) is always true since $G^{\prime}>0$; in this particular case, the theorem was proved in an entirely different manner by Petrini.*

* H. Petrini, Om ballistiska egenskaper hos kastbanor, A r k i v fö r M a t e m a t i k o c h F y s i k (Stockholm), vol. 7 (1912), pp. 1-29.

Trans. Am. Math. Soc. 24 . 
In actual practice, we have $v G^{\prime} / G>0.51$ and $h=0.0001036$, so that (71) is satisfied whenever the maximum ordinate does not exceed 7500 meters. In vacuum, the well-known formula $x_{\omega}=v_{0}^{2} \sin 2 \alpha / g$ shows that the range increases exactly as the square of the velocity.

From the first of (26) it follows, since $\lambda=1$ by (42) and $x^{\prime} \nu+y^{\prime} \rho>0$ by (65), that $\left(x^{\prime} \nu+x\right)^{\prime}>0$, so that $x^{\prime} \nu+x$ increases with $t$, and since $\nu=0$ at $t=t_{\omega}$ by (42), we find

$$
\nu<\frac{x_{\omega}-x}{x^{\prime}} \quad \text { for } \quad 0 \leqq t<t_{\omega} .
$$

Now (31) gives, by means of (68) and (73), since $\lambda=1$ and $x^{\prime}>0$,

$$
\left(x^{\prime} \rho-y^{\prime} \nu\right)^{\prime}<y^{\prime}-x^{\prime} \cot \omega+g \frac{x_{\omega}-x}{x^{\prime}} ;
$$

integrating from $t$ to $t_{\omega}$ and observing (42), we find

$$
\begin{aligned}
-\left(x^{\prime} \rho-y^{\prime} \nu\right)<\int_{t}^{t_{\omega}}\left[-x^{\prime} \cot \omega+y^{\prime}\right. & \left.+g \frac{x_{\omega}-x}{x^{\prime}}\right] d t \\
& =\left[-x \cot \omega-\left(x_{\omega}-x\right) \frac{y^{\prime}}{x^{\prime}}\right]_{t}^{t_{\omega}},
\end{aligned}
$$

the integration in finite terms being easily verified by means of (1), and finally

$$
x^{\prime} \rho-y^{\prime} \nu>\left(x_{\omega}-x\right)\left(\cot \omega-\frac{y^{\prime}}{x^{\prime}}\right) \text {. }
$$

Making $t=0$, and comparing to (48), we obtain for the range variation due to an increase in the angle of departure

$$
\delta x_{\omega}>x_{\omega}(\cot \omega-\tan \alpha) \delta \alpha,
$$

so that $\delta x_{\omega}>0$ when $\tan \alpha \leqq \cot \omega$ or $\alpha+\omega \leqq \pi / 2$; from the well-known fact that $\alpha<\omega$, it follows that

An increase in the angle of departure increases the range when the sum of the angles of departure and fall does not exceed $90^{\circ}$, or more particularly, when the angle of fall does not exceed $45^{\circ}$.

This theorem gives no information as to whether the maximum range at a given initial velocity occurs at an angle of departure equal to $45^{\circ}$ (which is the case in vacuum), or smaller or greater. In actual practice, all three cases are found to occur, according to the values of the initial velocity and the ballistic coefficient.* From (73), we obtain

$$
t_{\omega}-t_{0}-\nu\left(t_{0}\right)>t_{\omega}-t_{0}-\frac{x_{\omega}-x\left(t_{0}\right)}{x^{\prime}\left(t_{0}\right)},
$$

*A theoretical investigation of the special case of a homogeneous atmosphere $(h=0)$ and a retardation proportional to the $n$th power of the velocity is given by Petrini, l. c. 
and making $w_{x}=w_{z}$ in (49) and (19), it follows that

The increase in range due to a constant following wind from the time $t_{0}$ onward is greater than the deflection at the point of fall due to a constant cross wind of the same velocity from the same time $t_{0}$ onward.

Using (65) in (52), we see that

An increase in the ballistic coefficient increases the range.

Similarly it is shown that an increase in atmospheric density $(\delta H>0$ everywhere on the trajectory) or in retardation $(\delta G>0)$ will decrease the range.

\section{QUALITATIVE PROPERTIES OF THE DIFFERENTIAL VARIATIONS IN MAXIMUM ORDINATE*}

The fundamental inequality for the maximum ordinate variations is

$$
x^{\prime} \nu+y^{\prime} \rho>0 \quad \text { for } \quad 0 \leqq t<t_{s},
$$

and its proof is simpler than in the case of the range, since now $y^{\prime}$ does not change its sign in the time interval considered. Writing

$$
\psi=\int_{t}^{t_{*}}\left(1+\frac{v G^{\prime}}{G}\right) E d t
$$

we obtain from the second equation (54) and (55)

$$
e^{\psi}\left(x^{\prime} \nu+y^{\prime} \rho\right)=2 \int_{t}^{t_{\bullet}} e^{\psi} y^{\prime} \mu d t
$$

so that, since $y^{\prime}>0$ on the rising branch of the trajectory, and $\mu=1$ at $t=t_{s}, \mu$ is positive, and consequently also $x^{\prime} \nu+y^{\prime} \rho$ by (77), for $t$ less than but sufficiently close to $t_{s}$. If (76) is not true everywhere, there consequently exists a $t_{1}$ such that $x^{\prime} \nu+y^{\prime} \rho$ is zero at $t_{1}$, but positive for $t_{1}<t<t_{\text {a }}$. Then the first of (54) shows that $\mu$ decreases or is constant as $t$ increases from $t_{1}$ to $t_{\mathrm{s}}$ so that $\mu \geqq 1$ for $t_{1}<t<t_{\mathrm{s}}$ by (55), and (77) therefore gives a positive $x^{\prime} \nu+y^{\prime} \rho$ at $t=t_{1}$, contrary to our assumption. Thus (76) is proved. From the first of (54) and (76) it follows that $\mu^{\prime} \leqq 0$ so that $\mu$ decreases steadily toward unity as $t$ increases from 0 to $t_{\mathrm{s}}$ (or is constant $=1$ when $h$ vanishes identically). Using (76), the first of (26) shows, $\lambda$ being zero by (53)

* The following will explain why no discussion is given of the properties of the variations in time of flight, which are more important from a practical point of view than those in maximum ordinate. It follows from (39) that the specialization of (29) proper to the time of flight variations is $x=1, \lambda=0, \mu=-1 / y_{\nu}^{\prime}, \nu=\rho=0$ at $t=t_{\omega}$. The non-homogeneous terms in the second equation (29) then become $-\left(2 y^{\prime} \mu+1\right)$ taking the values +1 at tw and -1 at $t_{s}$. On account of this change of sign, the method used in the text to prove $x^{\prime} \nu+y^{\prime} p$ to be of constant sign is inapplicable. Moreover, numerical computation shows the behavior of the time of flight variations to be so much more complicated and irregular than in the cases of range and maximum ordinate, that it appears difficult even to formulate conjectures in respect to their qualitative properties. 
and (23), that $\left(x^{\prime} \nu\right)^{\prime}>0$, so that $x^{\prime} \nu$ increases toward the value zero at $t_{s}$, and consequently

$$
\nu<0 \quad \text { for } \quad 0 \leqq t<t_{s} \text {. }
$$

Since $y^{\prime}>0$, the comparison of (76) and (78) shows that

$$
\rho>0 \text { for } 0 \leqq t<t_{s},
$$

and the first of (54), (56) and (79) now give the inequality

$$
h y^{\prime} \mu+\mu^{\prime}=g \rho h \geqq 0,
$$

or by $(28)(\mu / H(y))^{\prime} \geqq 0$ whence, observing that $\mu=1$ at $t_{s}$,

$$
\mu \leqq \frac{H(y)}{H\left(y_{s}\right)},
$$

the equality sign holding only when $h$ is identically zero, or $H(y)=$ const. From (54) and (55), we find

$$
\begin{aligned}
x^{\prime} \nu+y^{\prime} \rho & =\int_{\iota}^{t_{s}}\left[2 y^{\prime} \mu-\left(1+\frac{v G^{\prime}}{G}\right) E\left(x^{\prime} \nu+y^{\prime} \rho\right)\right] d t \\
& =2\left(y_{s}-y \mu\right)-\int_{t}^{t_{s}}\left(1+\frac{v G^{\prime}}{G}-2 h y\right) E\left(x^{\prime} \nu+y^{\prime} \rho\right) d t
\end{aligned}
$$

and from (76) and (71) it is seen that for $t=0$ we have $0<\left(x^{\prime} \nu+y^{\prime} \rho\right)_{0}<2 y_{s}$, so that (58) gives for an increase $\delta v_{0}$ in initial velocity

$$
0<\frac{\delta y_{s}}{y_{s}}<2 \frac{\delta v_{0}}{v_{0}}
$$

or

For an increase in the initial velocity, the maximum ordinate increases, and for low trajectories, where $y$ is so small that (71) is satisfied everywhere, the maximum ordinate increases less rapidly than the square of the initial velocity.

When $h=0$, the latter part of the theorem is always true. Comparing (31) and (78), it is seen that

$$
\left(x^{\prime} \rho-y^{\prime} \nu\right)^{\prime}<-x^{\prime} \mu \leqq-x^{\prime}
$$

since $\mu \geqq 1$, whence integrating from $t$ to $t_{s}$

$$
x^{\prime} \rho-y^{\prime} \nu>x_{s}-x
$$

and making $t=0$ and using (59):

An increase in the angle of departure increases the maximum ordinate, the increase satisfying the inequality

$$
\delta y_{s}>x_{s} \delta \alpha
$$


The trajectory being concave downward, we evidently have $y_{s}<x_{s} \tan \alpha$, whence (83) gives

so that

$$
\frac{\delta y_{s}}{y_{s}}>\cot \alpha \delta \alpha=\frac{\delta(\sin \alpha)}{\sin \alpha}
$$

The maximum ordinate increases more rapidly than the sine of the angle of departure.

Incidentally, multiplying (76) by $y^{\prime},(82)$ by $x^{\prime}$ and adding, we find

$$
\rho>\frac{x^{\prime}\left(x_{s}-x\right)}{x^{\prime 2}+y^{\prime 2}}=\frac{x^{\prime}\left(x_{s}-x\right)}{v^{2}} .
$$

From (78) and (60) it is seen that

The maximum ordinate is increased by a constant following wind.

In the third of (26), all five factors in the second term to the right are positive, whence $\left(x^{\prime} \rho\right)^{\prime}>-x^{\prime} \mu$ and integrating from $t_{0}$ to $t_{s}$, where $t_{0}<t_{s}$, we find

$$
x^{\prime}\left(t_{0}\right) \rho\left(t_{0}\right)<\int_{t_{0}}^{t_{0}} x^{\prime} \mu d t,
$$

since $\rho\left(t_{s}\right)=0$ by (53), and consequently, since $\mu \geqq 1$,

$$
\begin{aligned}
\int_{t_{0}}^{t_{s}} \mu d t-\rho\left(t_{0}\right)>\int_{t_{0}}^{t_{0}} \mu\left[1-\frac{x^{\prime}}{x^{\prime}\left(t_{0}\right)}\right] d t \geqq \int_{t_{0}}^{t_{s}}\left[1-\frac{x^{\prime}}{x^{\prime}\left(t_{0}\right)}\right] d t & \\
& =t_{s}-t_{0}-\frac{x_{s}-x\left(t_{0}\right)}{x^{\prime}\left(t_{0}\right)} .
\end{aligned}
$$

Comparing (61) and (19), we therefore see that

The increase in maximum ordinate due to a constant vertical wind from the time $t_{0}$ onward is greater than the deflection at the summit due to a constant cross wind of the same velocity from the same time $t_{0}$ onward.

Using (76) in (63), we find that

An increase in the ballistic coefficient increases the maximum ordinate.

Similarly, it is shown that an increase in atmospheric density ( $\delta H>0$ everywhere on the trajectory) or in retardation $(\delta G>0)$ will decrease the maximum ordinate. We shall finally show that

The increase in maximum ordinate due to an increase in the ballistic coefficient satisfies the inequality

$$
\delta y_{s}>\left(2 y_{s}-x_{s} \tan \alpha\right) \frac{\delta C}{C}
$$

which is interesting inasmuch as the right hand member equals the (approximate) expression for $\delta y_{s}$ given by the Siacci theory.*

* See for instance Charbonnier, Balistique extérieure rationnelle, vol. 1 (Paris, Doin, 1907), p. 349 . 
To prove this, we observe that in (32), where now $\kappa=\lambda=0$, we have $y^{\prime}>0, x^{\prime} \nu+y^{\prime} \rho>0$ and $\mu \geqq 1$, whence

$$
\left[x^{\prime} E\left(x^{\prime} \nu+y^{\prime} \rho\right)\right]^{\prime}<-2 E x^{\prime} y^{\prime}
$$

and integrating from $t$ to $t_{s}$,

$x^{\prime} E\left(x^{\prime} \nu+y^{\prime} \rho\right)>\int_{t}^{t_{0}} 2 E x^{\prime} y^{\prime} d t=\left[-x^{\prime} y^{\prime}-g x\right]_{t}^{t_{0}}=x^{\prime} y^{\prime}-g\left(x_{s}-x\right)$,

the integration in finite terms being readily verified by means of (1). Consequently

$$
\begin{gathered}
E\left(x^{\prime} \nu+y^{\prime} \rho\right)>y^{\prime}-g \frac{x_{8}-x}{x^{\prime}} . \\
\int_{0}^{t_{0}} E\left(x^{\prime} \nu+y^{\prime} \rho\right) d t>\int_{0}^{t_{0}}\left(y^{\prime}-g \frac{x_{8}-x}{x^{\prime}}\right) d t \\
=\left[2 y+\left(x_{8}-x\right) \frac{y^{\prime}}{x^{\prime}}\right]_{0}^{t_{0}}=2 y_{8}-x_{8} \tan \alpha,
\end{gathered}
$$

and the substitution of this in (63) gives (85).

6. A special INTEgrable CASE, AND SOME CONJECtURes REgarding the RANGE VARIATIONS IN THE GENERAL CASE

The case to be considered is that of an atmosphere of constant density and a retardation proportional to the velocity, so that $h=0, G^{\prime}=0$. Here $\lambda, \mu, \nu, \rho$ may be expressed rationally in terms of $x, y, x^{\prime}$ and $y^{\prime}$. Considering the range variations only, for the sake of brevity, we have $\lambda=1$, the first equation (43) becomes $\mu^{\prime}=0$, so that $\mu=\cot \omega$ by (44), while the first and third of (26) become $\left(x^{\prime} \nu\right)^{\prime}=-x^{\prime}$ and $\left(x^{\prime} \rho\right)^{\prime}=-x^{\prime} \cot \omega$, so that, by (42),

$$
\nu=\frac{x_{\omega}-x}{x^{\prime}}, \quad \rho=\frac{x_{\omega}-x}{x^{\prime}} \cot \omega .
$$

From (45), it is now seen that

$$
E\left(x^{\prime} \nu+y^{\prime} \rho\right)=x^{\prime}+y^{\prime} \cot \omega-g \cot \omega \frac{x_{\omega}-x}{x^{\prime}},
$$

whence

$$
\begin{aligned}
\int_{0}^{t_{\omega}} E\left(x^{\prime} \nu+y^{\prime} \rho\right) d t & =\left[x+2 y \cot \omega+\cot \omega\left(x_{\omega}-x\right) \frac{y^{\prime}}{x^{\prime}}\right]_{0}^{c_{\omega}} \\
& =x_{\omega}(1-\cot \omega \tan \alpha) .
\end{aligned}
$$

Consequently, formulas (47) to (52) become 


$$
\begin{array}{ll}
\frac{\delta x_{\omega}}{x_{\omega}}=\left(1+\frac{\tan \alpha}{\tan \omega}\right) \frac{\delta v_{0}}{v_{0}} & \text { (initial velocity), } \\
\frac{\delta x_{\omega}}{x_{\omega}}=(\cot \omega-\tan \alpha) \delta \alpha & \\
\delta x_{\omega}=\left[t_{\omega}-t_{0}-\frac{x_{\omega}-x\left(t_{0}\right)}{x^{\prime}\left(t_{0}\right)}\right] w_{x} & \text { (angle of departure), } \\
\delta x_{\omega}=\left[t_{\omega}-t_{0}-\frac{x_{\omega}-x\left(t_{0}\right)}{x^{\prime}\left(t_{0}\right)}\right] \cot \omega \cdot w_{y} & \text { (vertical wind), } \\
\frac{\delta x_{\omega}}{x_{\omega}}=\left(1-\frac{\tan \alpha}{\tan \omega}\right) \frac{\delta C}{C} & \text { (ballistic coefficient). }
\end{array}
$$

These expressions lead to the following conjectures in the general case, which have been verified on a large number of computed trajectories, and which it would be very interesting to prove even under assumptions on $G(v)$ and $H(y)$ stronger than (64):

(a) The range variation due to an increase in initial velocity satisfies the inequality

$$
\frac{\delta x_{\omega}}{x_{\omega}}<\left(1+\frac{\tan \alpha}{\tan \omega}\right) \frac{\delta v_{0}}{v_{0}}
$$

(with or without the restriction (71)?).

(b) For $0 \leqq t<t_{\omega}, \nu$ is positive, so that, by (49), the range increase due to a constant following wind is less than what would be obtained by a rigid displacement of the original trajectory with the velocity of the wind during the time interval from $t_{0}$ to $t_{\omega}$.

(c) The range increase due to a constant vertical wind from the time $t_{0}$ onward is less than cot $\omega$ times the deflection at the point of fall due to a constant cross wind with the same velocity and from the same time $t_{0}$ onward.

(d) The range variation due to an increase in the ballistic coefficient satisfies the inequality

$$
\frac{\delta x_{\omega}}{x_{\omega}}>\left(1-\frac{\tan \alpha}{\tan \omega}\right) \frac{\delta C}{C},
$$

where the expression to the right is the one occurring in the Siacci theory (Charbonnier, l.c.).

Technical Staft,

Office of the Chief of Ordnance. 